\title{
Computer Aided Technique for Finger Print Image Enhancement and Minutiae Extraction
}

\author{
Rupali Telgad \\ Lecturer \\ MGM's Dr.G.Y.P.C.C.S.and IT \\ Aurangabad.
}

\author{
Prapti Deshmukh, PhD \\ Principal \\ MGM's Dr.G.Y.P.C.C.S.and IT \\ Aurangabad.
}

\begin{abstract}
Biometrics is the science and technology of measuring and analyzing biological data of human body, extracting a feature set from the acquired data, and comparing this set against to the template set in the database. Biometric techniques are gaining importance for personal authentication and identification as compared to the traditional authentication methods. User verification systems that use a single biometric indicator often have to contend with noisy sensor data, restricted degrees of freedom, and unacceptable error rates. To represent the large amount of data in the biometric images an efficient feature extraction method is needed. This paper presents the feature extraction of fingerprint image processing stages such as image Preprocessing, Converting the image in to gray scale, Image Enhancement can be performed with the help of Discrete Fourier transformation, image Binarization, image segmentation, image thinning, Minutiae Extraction after Minutiae Extraction again segmentation technique is used .In finger print images there are foreground regions and background regions where foreground region show the ridges and valleys while the background regions are to be left out. .The foreground regions have high value while the background regions have low values. Segmentation separates the foreground region from the background image for the reliable extraction of minutiae. The feature extraction is the first step used for matching used in biometric recognition. After feature extraction the next step is preprocessing of Minutiae. The extracted feature is called as templates, which is used in matching.
\end{abstract}

\section{Keywords}

Biometric systems, ridge, enhancement, finger print, FFT (Fast Fourier Transformation), ROI (Region of Interest)

\section{INTRODUCTION}

Biometric recognition involves recognition of biometric images that are deformed in degradations during the acquisition process due to factors such as imaging geometry, lens aberration, wrong focus, motion of scene and other random and systematic errors. Some of the challenges encountered by these systems are Noise in sensed data ${ }^{[1]}$. Image acquisition is the first step in the digital image processing; based on the mode of acquisition, a fingerprint image may be classified as off-line or live-scan. An off-line image is typically obtained by smearing ink on the fingertip and creating an inked impression of the fingertip on paper. A live-scan image, on the other hand, is acquired by sensing the tip of the finger directly, using a sensor that is capable of digitizing the fingerprint on contact. Live-scan is done using sensors. There are three basic types of sensors used. They are optical sensors, ultrasonic sensors and capacitance sensors ${ }^{[2]}$. Image enhancement is the process of manipulating the image so that the result is more suitable than the original image. Enhancement techniques use so many different image processing approaches that are difficult to assemble meaningful techniques suitable for the enhancement ${ }^{[3]}$. Fast Fourier transform (FFT) is one of the most fundamental operations in digital signal processing. Because of the efficiency of the convolution property, the FFT is often used in linear filtering found in many applications such as quantum mechanics, noise reduction and image reconstruction ${ }^{[4]}$. Morphological processing deals with tools for extracting image components that are useful in representation and description with the tools for extracting image components that are useful in representation and description with the help of shape of the image. It is used to represent the attribute of the image ${ }^{[3,5]}$ Segmentation procedure partition an image in to its constituent parts or objects to be identified individually.

\section{BIOMETRIC SYSTEM}

The term Biometric comes from the Greek word bios which mean life and metrikos which means measure. It is well known that humans intuitively use some body characteristics such as face, gait or voice to recognize each other. Since, a wide variety of application requires reliable verification schemes to confirm the ID of an individual, recognizing human on basis of their characteristics ${ }^{[6]}$. The characteristics are as follows:

1. Voice

2. Finger Prints

3. Body contours

4. Retina \& Iris

5. Face

6. Soft Biometrics.

A biometric system is fundamentally a pattern-recognition system that recognizes a individual based on a attribute vector derived from a specific physiological or behavioral characteristic that the person possesses. That feature vector is frequently stored in a database (or recorded on a smart card given to the individual) after being extracted. A biometric system based on physiological characteristics is normally more reliable than one which adopts behavioral characteristics, even if the last may be easier to integrate within certain specific application. Biometric system can than run in two modes: verification or identification. While recognition involves comparing the acquired biometric information against templates corresponding to all users in the database, verification involves comparison with only those templates corresponding to the claimed identity. This implies that identification and verification are two problems that should be deals with separately ${ }^{[7]}$.A simple biometric system 
consists of four basic components ${ }^{[8,9]}$ :a. Sensor module which acquires the biometric data.

b. Feature extraction module where the acquire data is processed to extract feature vectors.

c. Matching module where attribute vectors are compared against those in the template.

d. Decision-making module in which the user's identity is established or a claimed identity is accepted or rejected.

\subsection{Fingerprint Biometrics}

Fingerprints are unique for each finger of a person including identical twins. One of the most Instead; only a touch provides instant access. Fingerprint systems can also be used in identification mode ${ }^{[10]}$. The biometric fingerprint sensor takes a digital picture of a fingerprint. The fingerprint scan detects the ridges and valleys of a fingerprint and converts them into ones and zeroes. Complex algorithms analyze this raw biometric scan to identify characteristics of the fingerprint, known as the "minutiae". Minutiae are stored in a template, but only a subset of these has to match for identification or verification. The images acquired by these sensors are used by the feature extraction module to compute the feature values ${ }^{[11]}$. The feature values typically correspond to the position and orientation of certain critical points known as minutiae points (ridge endings and ridge bifurcations) that are present in every fingerprint (Figure.1).

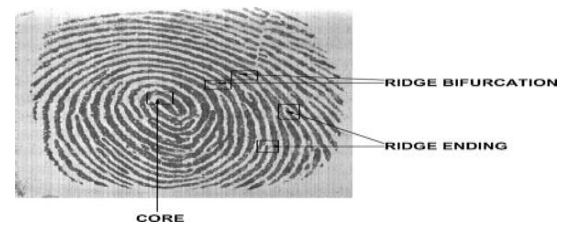

Figure 1: A fingerprint image with the core and four minutiae Points labeled

\section{PRAPOSED SYSTEMS:}

We propose system for an enhancement process, which combine filters and noise reduction techniques for pre and post processing. We use histogram equalization for contrast expansion and FFT for linear filtering which found in many applications such as quantum mechanics, noise reduction and image reconstruction. This is followed by the image binarization process. After image binarization image segmentation is performed on the images. Direction map of the images is the next step after image segmentation. Then ROI is considered for the next step. Thinning is then carried on the segmented Biometric image. Minutiae extraction is the next step used for Finger print recognition. The proposed system is as shown in the following Figure 2 as follows. The main stages of our proposed enhancement process conducted on the finger print image are shown in Figure 2 as follows.

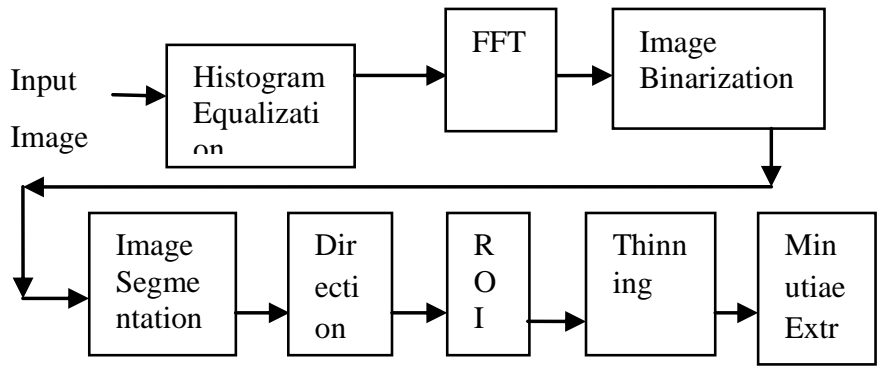

Figure 2: Proposed algorithm by adding the image segmentation

The proposed algorithm for the system is as shown in the following figure 3 .

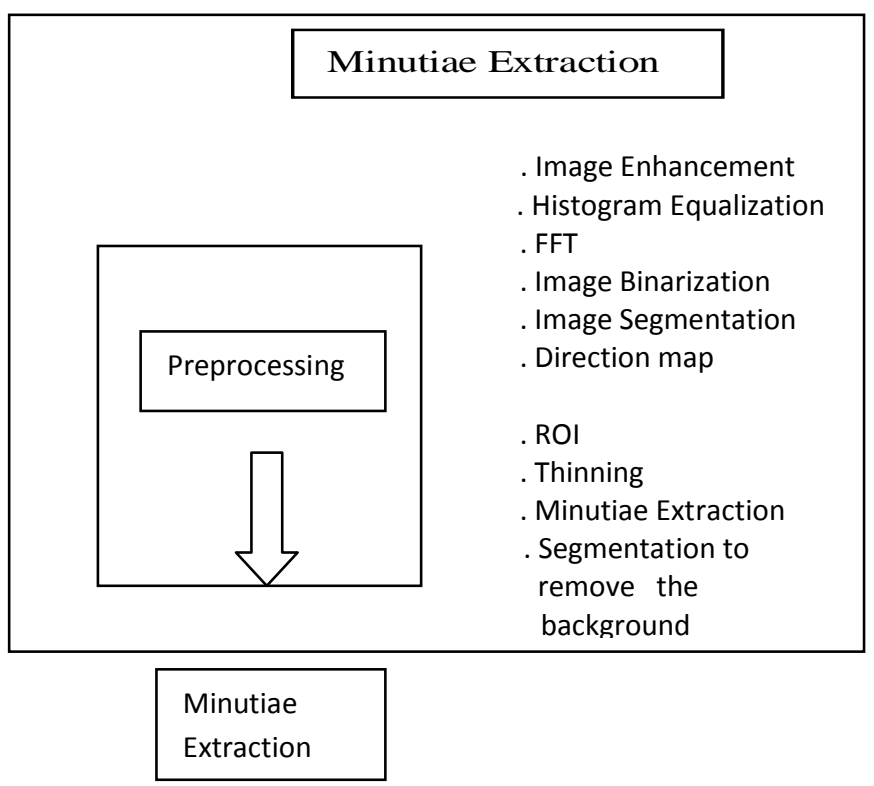

Figure 3: Minutiae Extraction with image segmentation (Designing the algorithm)

\section{FINGER PRINT ENHANCEMENT}

4.1 Data collection: For this paper presentation the database is taken from the FVC 2000 Fingerprint verification. The following figure shows the finger print image from the database which we used for the preprocessing and Minutiae Extraction.

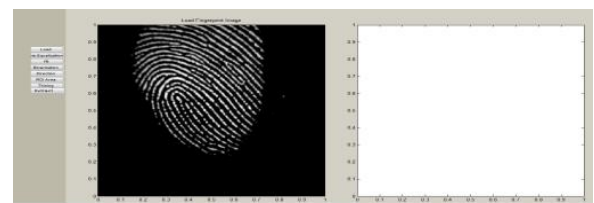

Figure 4: finger print image

\subsection{Finger print Image Enhancement}

Fingerprint image enhancement is used to make image clear for better use which is very easy to handle and can operate easily for further operation. The Image enhancement step is basically designed to reduce this noise and to enhance the definition of ridges against valleys ${ }^{[12]}$. In this paper we use the following image enhancement stages those are-

1) Histogram Equalization:

Fast Fourier Transformation.

2) Binarization

\subsubsection{Histogram Equalization:}

The transformation generates an image whose intensity levels are equal and covers range ${ }^{[0,1]}$. The result of this intensity levels equalization process is an image with increased dynamic range, which will tend to have high contrast. The transformation function is not more than 
cumulative distribution function (CDF). For the discrete we work with the summations, and equalization transformation becomes

$$
S k=T(r K)
$$

Where $\mathrm{Sk}$ is the intensity value in the output image corresponding to value $\mathrm{T}(\mathrm{rK})$ in the input image ${ }^{[13]}$. The right side of the following figure 5 is the output after the histogram equalization.

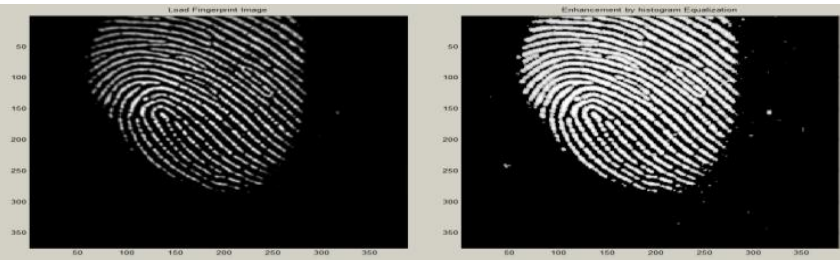

Figure 5: Histogram equalization

\subsubsection{Fast Fourier Transformation:}

All filtering is carried out in a frequency domain through the Fourier transform. In this paper we use Fast Fourier Transformation on the Biometric images. Here first of all we divide the image into different small processing blocks then use the Fourier transform according to formula:

$$
F(u, v)=\sum_{x=0}^{M-1 N-1} \sum_{y=0} f(x, y) \times \exp \left\{-j 2 \pi \times\left(\frac{u x}{M}+\frac{v y}{N}\right)\right\}
$$

for $\mathrm{u}=0,1,2, \ldots, 31$ and $\mathrm{v}=0,1,2, \ldots, 31$.

In order to enhance a specific block by its dominant frequencies, we multiply the FFT of the block by its magnitude a set of times. Where the magnitude of the original $\mathrm{FFT}=\operatorname{abs}(\mathrm{F}(\mathrm{u}, \mathrm{v}))=|\mathrm{F}(\mathrm{u}, \mathrm{v})|$.Given $\mathrm{F}(\mathrm{u}, \mathrm{v})$ we obtained from $\mathrm{F}(\mathrm{x}, \mathrm{y})$ through the inverse Fourier transform given by the expression.

$$
f(x, y)=\frac{1}{M N} \sum_{x=0}^{M-1} \sum_{y=0}^{N-1} F(u, v) \times \exp \left\{j 2 \pi \times\left(\frac{u x}{M}+\frac{v y}{N}\right)\right\}
$$

for $\mathrm{x}=0,1, \ldots \mathrm{M}-1$ and $\mathrm{y}=0,1,2, \ldots ., \mathrm{N}-1$.equation 1 and equation 2 comprise the two dimensional, FFT pair. The variables $\mathrm{u}$ and $\mathrm{v}$ are the transform or frequency variables and $x, y$ are the spatial or image variables ${ }^{[3]}$. The enhanced image after FFT has the improvements to connect some falsely broken points on ridges and to remove some spurious connections between ridges. The shown image at the left side of Figure 6 is also processed with histogram equalization after the FFT transform.

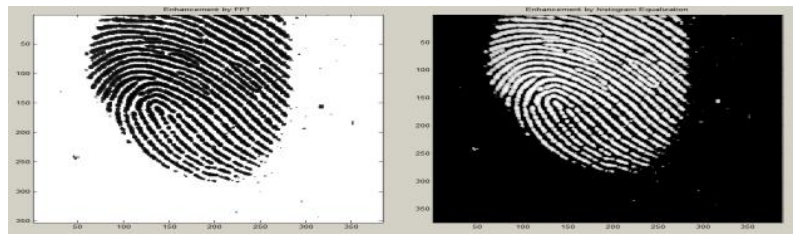

Figure 6 Fast Fourier Transformations

\subsubsection{Fingerprint image binarization:}

Image binarization is the process of turning a gray scale image to a black and white image. By the end of this process, all pixel values within the image are either zero or one, and the image has been converted to binary format ${ }^{[15]}$.Fingerprint Image Binarization is used to transform the 8-bit Gray fingerprint image to a 1- bit image and here the value for the ridges is 0 where as it is 1 for the furrows. After this operation, the ridges in the fingerprint will be highlighted with black color while some with white ${ }^{[16]}$. A locally adaptive binarization method is performed to binaries the fingerprint image. Such a named method comes from the mechanism of transforming a pixel value to 1 if the value is larger than the mean intensity value of the current block $(16 \times 16)$ to which the pixel belongs [Figure 7].
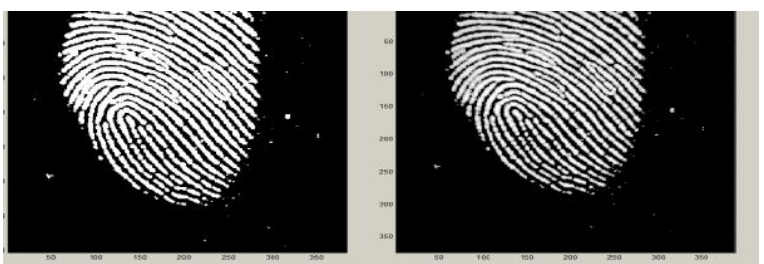

Figure 7: Finger print Binarization

4.3 Image segmentation: Segmentation divides the image in to its constituent regions or objects. Segmentation stops when the object or region of interest in an application has been detected. In image analysis if some part of the object anomalies, such as missing component is their then segmentation required to identify those elements ${ }^{[3]}$. In a fingerprint image there are foreground regions and the background regions. The foreground regions show the ridges and valleys while the background regions are to be left out. The foreground regions have a high variance value while the background regions have low values. Segmentation separates the foreground regions from the background image for reliable extraction of minutiae 12 . We add image segmentation algorithm in the system which remove the background of the image. The right hand side of the following figure 8 shows the segmentation followed by Binarization of the image.
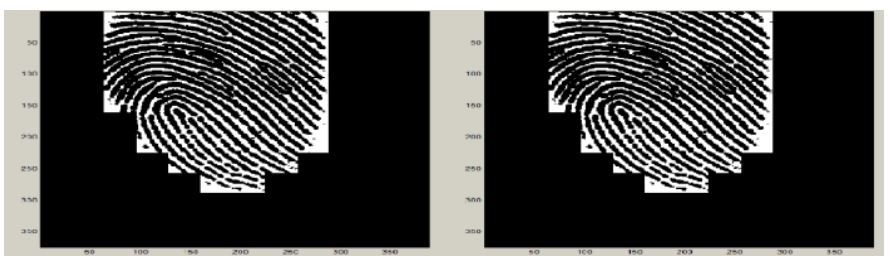

Figure 8: After segmentation

\subsection{Direction Map:}

Estimate the block direction for each block of the fingerprint image with $\mathrm{Wx} \mathrm{W}$ in size ( $\mathrm{W}$ is 16 pixels by default). The algorithm is used to calculate the gradient values along $\mathrm{x}$ direction (gx) and y-direction (gy) for each pixel of the block. The direction map is as shown in the following figure 9 to right.

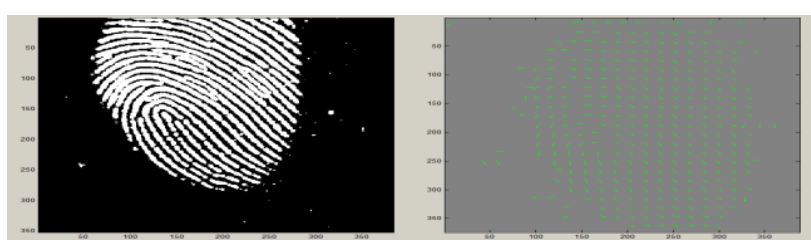

Figure 9: Direction map

\subsection{ROI:}

Figure 10 show the interest fingerprint image area and it's bound. The bound is the subtraction of the closed area from 
the opened area. Then the algorithm throws away those leftmost, rightmost, uppermost and bottommost blocks out of the bound so as to get the tightly bounded region just containing the bound and inner area.
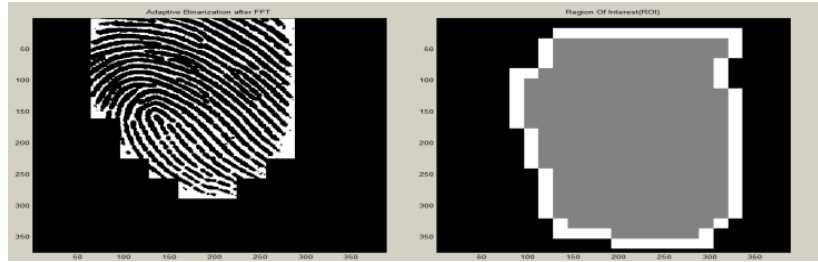

Figure 10: ROI

\section{6: Finger Print Ridge Thinning:}

Ridge Thinning is to eliminate the redundant pixels of ridges till the ridges are just one pixel wide. ${ }^{[1]}$ Uses an iterative, parallel thinning algorithm. In each scan of the full fingerprint image, the algorithm marks down redundant pixels in each small image window $(3 \times 3)$. And finally removes all those marked pixels after several scans. In my testing, such an iterative, parallel thinning algorithm has bad efficiency although it can get an ideal thinned ridge map after enough scans. Their method traces along the ridges having maximum gray intensity value. The Following right hand side figure 11 shows the finger print Ridge Thinning.
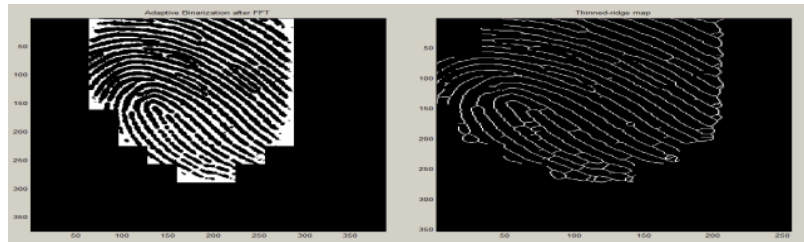

Figure 11: Thinning

4.7 Finger print minutiae Detection: For minutiae detection we use the cross numbering algorithm. After the fingerprint ridge thinning, marking minutia points is relatively easy. ${ }^{[22]}$ uses for each $3 \times 3$ window, if the central pixel is 1 and has exactly 3 one-value neighbors, then the central pixel is a ridge branch [Figure 12]. If the central pixel is 1 and has only 1 one-value neighbor, then the central pixel is a ridge ending [Figure 13].

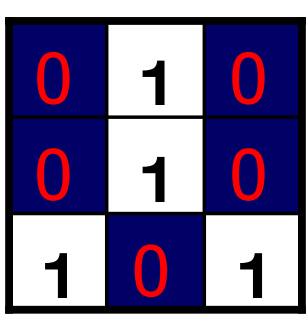

Figure12: Bifurcation

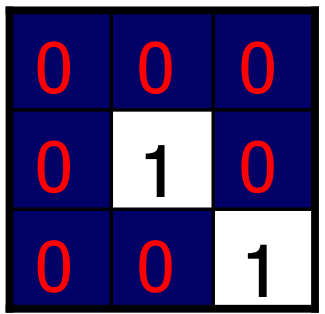

Figure 13: Termination
Together with the minutia marking, all thinned ridges in the fingerprint image are labeled with a unique ID for further operation. The labeling operation is realized by using the Morphological operation: BWLABEL. Then we implement the cross numbering algorithm to extract the Minutiae from the thinned image. The right hand side shows the minutiae extraction of the finger print image.
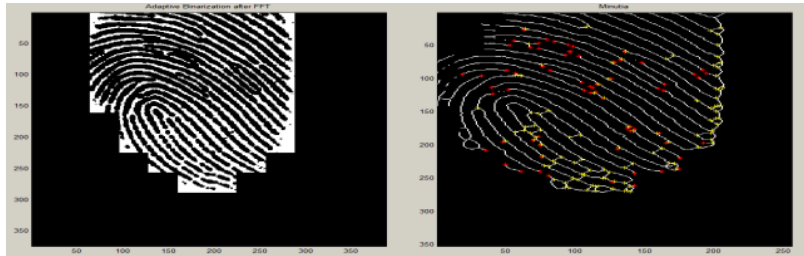

Figure 15: Finger print minutiae extraction

We developed the system with the help of segmentation algorithm which segments the image after binarization as well as after the minutiae detection. The segmentation algorithm removes the Background and it only detects the Minutiae points. The following figure 16 shows the minutiae detection after segmentation.
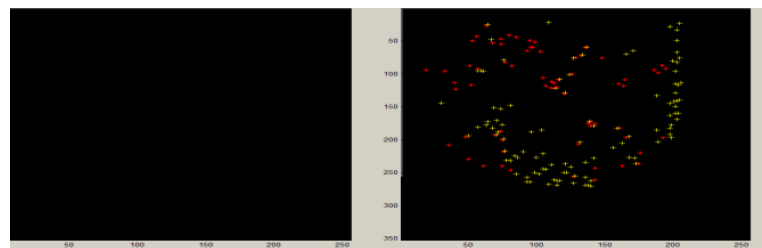

Figure 16: Finger print minutiae extraction after background removal

5. Experimental Results: The Experiment is carried on the 10 images. The following table 1 shows the implementation of various steps of the proposed algorithm from Input image to the Minutiae Extraction.

6. Conclusions: Many business applications will in future rely on biometrics since using biometrics is the only way to guarantee the presence of the owner when a transaction is made. Finger print recognition systems have been proven to be very effective in protecting information and resources in a large area of applications. We implement Biometric fingerprint feature extraction system is via Image enhancement, Image Binarization, Image segmentation, Image thinning, Direction map, ROI, Minutiae detection techniques by using cross numbering algorithm and again we use segmentation to only extract the minutiae points. In this paper we used three techniques for Image Enhancement 1) Histogram Equalization 2) Fast Fourier Transformation 3) Binarization. In this paper we developed our own system in which FFT is used for removing noise as well as thereconstruction. Binarization is used for image enhancement. Image segmentation is used to remove the background .Various standard techniques are used in the intermediate stages of processing. These steps are as shown in the following table 
Table 1: Practical Implementation

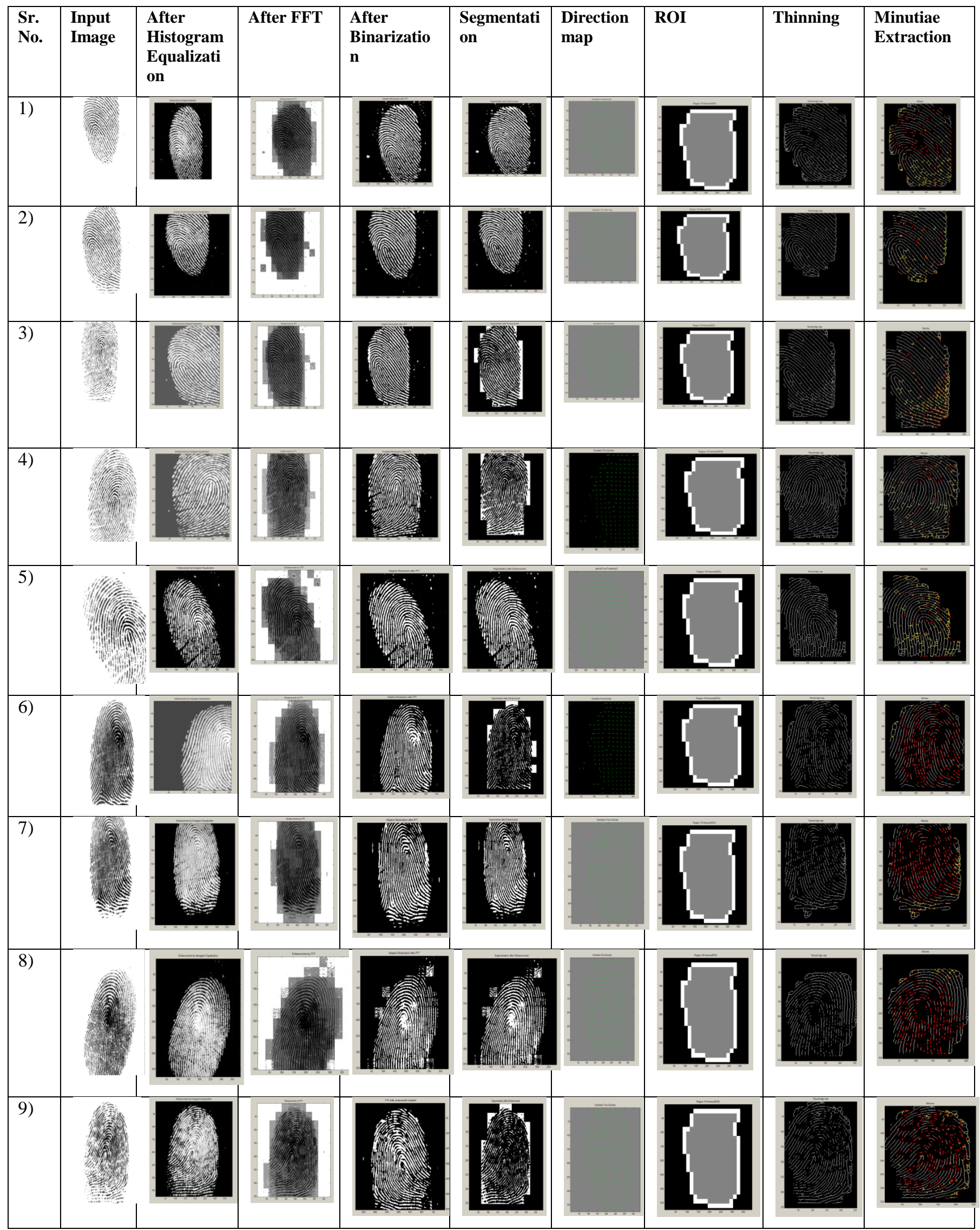




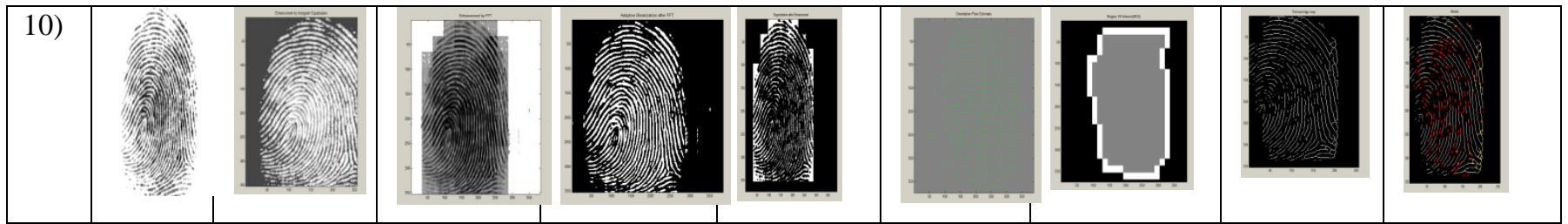

The Following table 2 shows the std. deviation calculated for the input images as well as the minutiae Extracted images.

Table 2 : Standard Deviation

\begin{tabular}{|c|c|}
\hline $\begin{array}{l}\text { Std. Dev. For Input } \\
\text { image }\end{array}$ & $\begin{array}{l}\text { Std. deviation for Minutiae } \\
\text { Extraction }\end{array}$ \\
\hline 52.595 & 96.9689 \\
\hline 52.2941 & 91.4776 \\
\hline 52.9723 & 97.4631 \\
\hline 57.6061 & 98.2332 \\
\hline 56.12199 & 94.0075 \\
\hline 86.6304 & 95.7076 \\
\hline 81.0535 & 91.5222 \\
\hline 78.3703 & 93.5963 \\
\hline 67.8216 & 95.5805 \\
\hline 63.8675 & 94.4936 \\
\hline
\end{tabular}

The Following graph shows the quality of the image is improved while minutiae extraction as the std. deviation is increased.

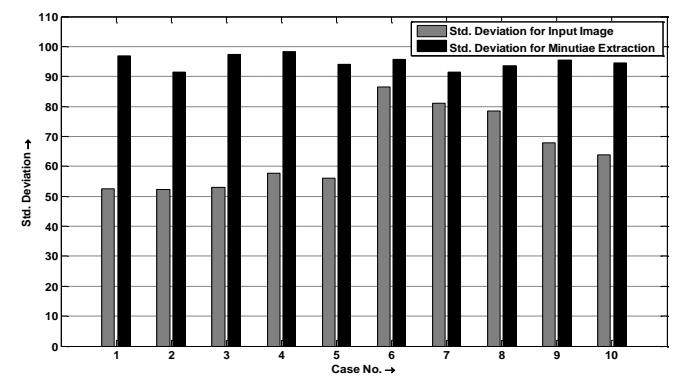

Figure 17: Statistical Analysis

\section{Acknowledgements:}

We are thankful to our Guide Dr. P. D. Deshmukh for providing valuable guidance and technical support.

\section{REFERENCES}

[1] WUZHILI, "Finger print recognition “,Computer System Major Department of Computer Science Hong Kong Baptist University.

[2] SHOUGAIJAM DEBAJIT SINGH, SHIBA PRASAD MAJHI,"Fingerprint Recognition: A study on image enhancement and minutia extraction".

[3] Rafael C. Gonzalez, Richard E. Woods, "Digital image processing", Third Edition, Pearson Publication.
[4] J.S. Walker, Fast Fourier Transforms, CRC Press, $2^{\text {nd }}$ edition, 1996.

[5] Dakshina Ranjan Kisku1, Phalguni Gupta2, Jamuna Kanta Sing3,"Multibiometrics Feature level fusion by Graph clustering", International Journal of Security and Its Applications Vol. 5 No. 2, April, 2011

[6] Neena Godbole, "Information Security System”, Wiley Publication.

[7] Khattab M. Ali Alheeti, "Biometric Iris Recognition Based on Hybrid Technique", International Journal on Soft Computing ( IJSC ) Vol.2, No.4, November 2011.

[8] 6th International Symposium Electronics in Marine, ELMAR-2004, 16-18 June 2004, Zadar, Croatia 185.

[9] S. Prabhakar, S. Pankanti, A. K. Jain, "Biometric Recognition: Security and Privacy Concerns", IEEE Security \& Privacy, March/April 2003, pp. 33-42.

[10] Olufemi Sunday Adeoye, "A Survey of Emerging Biometric Technologies", International Journal of Computer Applications (0975 - 8887) Volume 9- No.10, November 2010.

[11] Arun Ross, Anil Jain, "Information fusion in biometrics", Department of Computer Science and Engineering, Michigan State University, 3115 Engineering Building, East Lansing, MI48824, USA

[12] TATSAT NAIK And OM SRI SATYASAI Under the Guidance of Prof. S. MEHER,'Study of finger prints recognition system"

[13] Rafeal C. Gonzalez, Richard E. Woods, Steven L. Eddins,"Digital Image Processing Using Matlab" Mc Graw Hill

[14] Jianwei Yang, Lifeng Liu, and Tianzi Jiang, "An Improved Method for Extraction of Fingerprint Features", National Laboratory of Pattern Recognition, Institute of Automation, Chinese Academy of Sciences, Beijing 100080, P. R. China.

[15] Kondreddi Gopi, J.T Pramod, 'Fingerprint Recognition Using Gabor Filter And Frequency Domain Filtering" IOSR Journal of Electronics and Communication Engineering (IOSRJECE) ISSN: 2278-2834 Volume 2, Issue 6 (Sep-Oct 2012), PP 17-21

[16] TATSAT NAIK and OM SRI SATYASAI under the Guidance of Prof. S. MEHER "Study of fingerprint recognition system",

[17] Feng Zhao," A Brief Introduction to Skeleton-Based Fingerprint Minutiae Extraction", School of Computer Engineering, Nanyang Technological University, Singapore 639798 Rutovitz D. Pattern recognition [J]. J. Roy. Stat. Soc., 1966, 129: 504-530 
[18] Hayit Greenspan, Charles H. Anderson, and Sofia Akber, "Image Enhancement By Nonlinear Extrapolation in Frequency Space", IEEE TRANSACTIONS ON IMAGE PROCESSING, VOL. 9, NO. 6, JUNE 2000

[19] Sonam Shukla, Pradeep Mishra "Increasing The Accuracy Of An Existing Fingerprint Recognition System Using Adaptive Technique". International Journal of Advanced Research in Computer Science and Software Engineering
[20] L.C. Jain, U.Halici, I. Hayashi, S.B. Lee and S.Tsutsui. "Intelligent biometric techniques in fingerprint and face recognition". 1999, the CRC Press.

[21] C. Lakshmi Deepika, Dr. A Kandaswamy, C. Vimal, and B. Sathish,"Invariant Feature Extraction from Fingerprint Biometric Using Pseudo Zernike Moments", International Journal of Computer Communication and Information System (IJCCIS) Vol2. No1. ISSN: 09761349 July - Dec 2010 